\title{
Symmetry-Breaking under Small Perturbations
}

\author{
K.-S. Cheng \& J. A. SMOller \\ Communicated by J. SERRIN
}

\section{§ 1. Introduction}

Consider the Dirichlet problem

$$
\begin{aligned}
\Delta u(x)+f(u(x)) & =0, & & x \in D_{R}^{n} \\
u(x) & =0, & & x \in \partial D_{R}^{n} .
\end{aligned}
$$

Here $f$ is a smooth function, and $D_{R}^{n}(n \geqq 2)$ is the open ball of radius $R$ in $\mathbb{R}^{n}$, centered at the origin. In an interesting paper GIDAS, NI, \& NrRENBERG [GNN] proved that positive solutions of (1.1) must be spherically symmetric; i.e., $u$ must be a radial function, $u=u(r), r=|x|$. Thus $u(r)$ satisfies the ordinary differential equation

$$
\begin{gathered}
u^{\prime \prime}+\frac{n-1}{r} u^{\prime}+f(u)=0, \quad 0<r<R \\
u^{\prime}(0)=0=u(R) .
\end{gathered}
$$

Recently, SMOLLER \& WASSERMAN [SW1, 2] considered the bifurcation problem for positive radial solutions. Specifically, they investigated the ways in which a symmetric solution can bifurcate into an asymmetric solution. Of course, the results of [GNN] imply that these asymmetric solutions cannot be positive functions.

It is natural to allow the function $f$ to depend on both $u$ and $|x|$, and to consider the Dirichlet problem

$$
\begin{aligned}
\Delta u(x)+f(u(x),|x|) & =0, \quad x \in D_{R}^{n}, \\
u(x) & =0, \quad x \in \partial D_{R}^{n} .
\end{aligned}
$$

It was shown in [GNN], that if $f_{r}(u, r) \leqq 0$ on the relevant range of $u$ and $r$, then positive solutions of (1.3) again must be radial functions. It is thus quite interesting to investigate the bifurcation problem for positive solutions of (1.3). We remark however that the general problem (1.3) is quite difficult to study, 
even for the existence of radial solutions. We shall therefore only consider small perturbations of (1.1), i.e., we study the problem

$$
\begin{aligned}
\Delta u(x)+f(u(x))+\varepsilon h(u(x),|x|)) & =0, \quad x \in D_{R}^{n} \\
u(x) & =0, \quad x \in \partial D_{R}^{n},
\end{aligned}
$$

Here $f$ and $h$ are smooth functions of their arguments, $\varepsilon$ is a small parameter, and we shall assume that $f$ satisfies the same conditions as in [SW2]; see $\S 2$.

In $\S 3$ we shall prove the existence of positive radial solutions of (1.4) for small $\varepsilon$. Furthermore, in preparation for our bifurcation results, we shall prove the existence of "degenerate" radial solutions; i.e., solutions having the property that zero lies in the spectrum of their linearizations. We consider the symmetrybreaking problem in $\S 4$. We also show there that if $h_{r}>0$, then there exist positive asymmetric solutions of (1.4); thus the result in [GNN] is sharp. Finally, in $\S 5$ we shall discuss a special application of our results to a weakly coupled system of equations.

\section{§ 2. Background Results}

We consider the following initial-value problem for positive radial solutions of $(1.1)$ :

$$
\begin{array}{r}
u^{\prime \prime}(r)+\frac{n-1}{r} u^{\prime}(r)+f(u(r))=0 \\
u(0)=p>0, \quad u^{\prime}(0)=0 .
\end{array}
$$

We denote by $u(r, p)$ the unique solution of $(2.1)$; as in [SW1, 2], $p$ will be considered a parameter. It was shown in [SW2], that if $f$ satisfies the conditions

$$
f(0)<0, \quad(f(u) / u)^{\prime}>0, \quad f^{\prime \prime}(u) \leqq 0,
$$

then for generic $f$, there exists a $\bar{p}>0$ and a function $T:[\bar{p}, \infty) \rightarrow \mathbb{R}$ such that for $p>\bar{p}, u(r, p)$ satisfies the following:

$$
\begin{gathered}
u(r, p)>0 \quad \text { if } \quad 0 \leqq r<T(p), \quad u(T(p), p)=0, \\
u^{\prime}(r, p)<0 \quad \text { if } \quad 0<r \leqq T(p) .
\end{gathered}
$$

Moreover, $u(r, \bar{p})$ satisfies

$$
\begin{array}{ccc}
u(r, \bar{p})>0: & \text { if } 0 \leqq r<T(\bar{p}), & u(T(\bar{p}), \bar{p})=0 \\
u^{\prime}(r, \bar{p})<0 & \text { if } 0<r<T(\bar{p}), & u^{\prime}(T(\bar{p}), \bar{p})=0 \\
& u_{p}(T(\bar{p}), \bar{p})<0
\end{array}
$$

Thus, if we consider the equation

$$
u(R, p)=0,
$$

then we can solve for $p$ as a function of $R$ in a neighborhood of the point $(T(\bar{p}), \bar{p})$. Let $\bar{R}=T(\bar{p})$; then $p(R)$ is defined in an interval $\left[R_{1}, R_{2}\right]$ containing $\bar{R}$. From 
(2.5), we have, on this interval

and

$$
u^{\prime}(R, p(R))+u_{p}(R, p(R)) p^{\prime}(R)=0
$$

$u^{\prime \prime}(R, p(R))+2 u_{p}^{\prime}(R, p(R)) p^{\prime}(R)+u_{p}(R, p(R)) p^{\prime \prime}(R)+u_{p p}(R, p(R))\left(p^{\prime}(R)\right)^{2}=0$.

At $R=\bar{R},(2.6)$ gives $u_{p}(\bar{R}, p(\bar{R})) p^{\prime}(\bar{R})=0$, and since $u_{p}(\bar{R}, p(\bar{R}))<0$, we get

Thus from (2.7),

$$
p^{\prime}(\bar{R})=0
$$

$$
u^{\prime \prime}(\bar{R}, p(\bar{R}))+u_{p}(\bar{R}, p(\bar{R})) p^{\prime \prime}(\bar{R})=0
$$

Now from (2.1),

thus

$$
\begin{aligned}
u^{\prime \prime}(\bar{R}, p(\bar{R})) & =-\frac{n-1}{\bar{R}} u^{\prime}(\bar{R}, p(\bar{R}))-f u(\bar{R}, p(\vec{R})) \\
& =-f(0)
\end{aligned}
$$

$$
p^{\prime \prime}(\bar{R})=f(0) / u_{p}(\bar{R}, p(\bar{R}))>0 .
$$

Furthermore, for $R_{1} \leqq R<\bar{R}, u(R, p(R))$ satisfies

$$
\begin{array}{ll}
u(r, p(R))>0, & 0 \leqq r<R, \quad u(R, p(R))=0 \\
u^{\prime}(r, p(R))<0, & 0<r \leqq R .
\end{array}
$$

For $R=\bar{R}, u(r, p(\vec{R}))$ satisfies

$$
\begin{array}{ccc}
u(r, p(\bar{R}))>0, & 0 \leqq r<\bar{R}, & u(\bar{R}, p(\bar{R}))=0, \\
u^{\prime}(r, p(\bar{R}))<0, & 0<r<\bar{R}, & u^{\prime}(\bar{R}, p(\bar{R}))=0 .
\end{array}
$$

Finally, for $\bar{R}<R \leqq R_{2}, u(r, p(\ddot{T}))$ satisfies

$$
\begin{array}{rrrr}
u(r, p(R))>0, & 0 \leqq r<\tilde{R}(R), & u(\tilde{R}(R), p(R))=0, \\
u(r, p(R))<0, & \tilde{R}(R)<r<R, & u(R, p(R))=0, \\
u^{\prime}(r, p(R))<0, & 0<r<\tilde{R}(R), & u^{\prime}(\tilde{\tilde{R}}(R), p(R))=0, \\
u^{\prime}(r, p(R))>0, & \tilde{\tilde{R}}(R)<r \leqq R, &
\end{array}
$$

where $\tilde{R}(r)$ and $\tilde{\tilde{R}}(R)>\tilde{R}(R)$ are two smooth functions of $R$.

We say that a solution $u$ of $(1.1)$ is non-degenerate provided that $v \equiv 0$ is the only solution of the linearized equations

$$
\begin{aligned}
\Delta v(x)+f^{\prime}(u(x)) v(x)=0, & x \in D_{R}^{n}, \\
v(x) & =0, \quad x \in \partial D_{R}^{n} .
\end{aligned}
$$


Thus $u$ is non-degenerate if and only if zero is not in the spectrum of the associated linearized operator; otherwise $u$ is called degenerate. The following theorem was proved in [SW1]; we shall sketch the proof for the convenience of the reader, since the techniques in the proof will be used below.

Theorem 2.1. A. Assume $R_{1} \leqq R<\bar{R}$, and let $u(\cdot, p(R))$ be the corresponding radial solution of (1.1). Then $u$ is non-degenerate.

B. The solution $u(r, p(\bar{R}))$ is degenerate and the function $v(r, \theta)=u^{\prime}(r, p(\bar{R}))$ $\Phi_{1}(\theta)$ solves $(2.13) ; \Phi_{1}$ lies in the first eigenspace of the Laplacian on $S^{n-1}$, corresponding to the eigenvalue $\lambda_{1}=-(n-1)$.

Proof. Every solution of (2.13) for $u=u(;, p(R))$ can be written in its spherical harmonic decomposition

$$
v(r, \theta)=\sum_{N=0}^{\infty} a_{N}(r) \Phi_{N}(\theta), \quad \theta \in S^{n-1}, \quad 0 \leqq r \leqq R,
$$

where $\Phi_{N}$ belongs to the $N^{\text {th }}$ eigenspace of the Laplacian on $S^{n-1}$, corresponding to the eigenvalue $\lambda_{N}=-N(N+n-2), N=0,1,2, \ldots$ Using this representation in (2.13), we have, for $N \geqq 0$, that $a_{N}$ satisfies the differential equation

$$
a_{N}^{\prime \prime}+\frac{n-1}{r} a_{N}^{\prime}+\left(\frac{\lambda_{N}}{r^{2}}+f^{\prime}(u)\right) a_{N}=0,
$$

and if $N \geqq 1, a_{N}$ satisfies the boundary conditions

while for $N=0$,

$$
a_{N}(R)=0=a_{N}(0),
$$

$$
a_{0}^{\prime}(0)=0=a_{0}(R) \text {. }
$$

We begin by proving $a_{0}(r) \equiv 0$. First, $a_{0}(r)$ satisfies

$$
a_{0}^{\prime \prime}+\frac{n-1}{r} a_{0}^{\prime}+f^{\prime}(u) a_{0}=0 .
$$

Also $w(r) \equiv u_{p}(r, p(R))$ satisfies

$$
\begin{gathered}
w^{\prime \prime}+\frac{n-1}{r} w^{\prime}+f^{\prime}(u) w=0, \\
w^{\prime}(0)=0, \quad w(0)=1, \quad w(R)<0 .
\end{gathered}
$$

Comparing (2.15) and (2.16) gives $a_{0}(r)=a_{0}(0) w(r)$, so that $0=a_{0}(R)=$ $a_{0}(0) w(R)$ implies $a_{0}(0)=0$ and thus $a_{0}(r) \equiv 0$. Next we show $a_{N}(r) \equiv 0$ if $N \geqq 1$. If we let $z(r)=u^{\prime}(r, p(R))$, then $z$ satisfies

$$
\begin{gathered}
z^{\prime \prime}+\frac{n-1}{r} z^{\prime}+\left(\frac{\lambda_{1}}{r^{2}}+f^{\prime}(u)\right) z=0, \quad 0<r<R \\
z(0)=0, \quad z(r)<0, \quad 0<r \leqq R .
\end{gathered}
$$


Then multiplying (2.14) by $r^{n-1} z$ and (2.17) by $r^{n-1} a_{N}$ gives

$$
\frac{d}{d r}\left[r^{n-1}\left(a_{N}^{\prime} z-a_{N} z^{\prime}\right)\right]=r^{n-3}\left(\lambda_{1}-\lambda_{N}\right) a_{N} z
$$

Now assume that $N \geqq 2, a_{N}(r) \neq 0$, and that $\hat{R} \leqq R$ is the first positive zero of $a_{N}$; then $a_{N}(r)<0$ for $0<r<\hat{R}, a_{N}(\hat{R})=0$ and $a_{N}^{\prime}(\hat{R}) \geqq 0$. Integrating (2.18) from 0 to $\hat{R}$ gives the contradiction

$$
0 \geqq \hat{R}^{n-1} a_{N}^{\prime}(\hat{R}) z(\hat{R})=\int_{0}^{\hat{R}} r^{n-3}\left(\lambda_{1}-\lambda_{N}\right) a_{N} z d r>0 .
$$

Thus $a_{N}(r) \equiv 0$ if $N \geqq 2$. For $N=1$, we integrate (2.18) from 0 to $R$ to get $R^{n-1} a_{1}^{\prime}(R) z(R)=0$ so that $a_{1}^{\prime}(R)=0$. Thus $a_{1} \equiv 0$, and this completes the proof of (A).

In order to prove (B), we observe that in part (A). if $v(r, \theta)=\sum_{N \geq 0} a_{N}(r) \Phi_{N}(\theta)$, then the same arguments yield $a_{0}(r) \equiv 0$, and $a_{N}(r) \equiv 0$ if $N>1$. Moreover, it is easy to see that $a_{1}(r)=u^{\prime}(r, p(\bar{R}))$ solves $(2.14)$ and $a_{1}(R)=a_{1}(0)=0$. Thus (B) holds and the proof is complete.

Our next result deals with the solutions $u(r, p(R))$ in the range $\bar{R}<R<R_{2}$.

Theorem 2.2. Let $\bar{R}<R<R_{2}$; then the solution $u(r, p(R))$ is non-degenerate provided that $\left(R_{2}-\bar{R}\right)$ is sufficient small.

Proof. For $u=u(r, p(R)), \quad \bar{R}<R<R_{2}$, we use the expansion $v=$ $\sum_{N \geqq 0} a_{N}(r) \Phi_{N}(\theta)$, as in the previous theorem. For $\left(R_{2}-\bar{R}\right)$ small, we again have $u_{p}(R, p(R))<0$ so that the same arguments as in the proof of the last theorem (Part A) apply to give $a_{0}(r) \equiv 0$. Moreover, as $u^{\prime}(R, p(R))>0$ (by (2.12)), we conclude, as in the proof of Part A of the last theorem, that $a_{1}(r) \equiv 0$.

Now for $N \geqq 2$, we have, as before

$$
\frac{d}{d r}\left[r^{n-1}\left(a_{N}^{\prime} z-a_{N} z^{\prime}\right)\right]=r^{n-3}\left(\lambda_{1}-\lambda_{N}\right) a_{N} z
$$

where $z(r)=u^{\prime}(r, p(R))$. Again assume that $a_{N}(r) \neq 0$ and that $\hat{R} \leqq R$ is the first positive zero of $a_{N}$. We may assume that $a_{N}(r)<0$ if $0<r<\hat{R}$. Then if $\hat{R} \leqq \widetilde{\widetilde{R}}(c f .(2.13))$, we integrate $(2.20)$ from 0 to $\hat{R}$ to get

$$
\hat{R}^{n-1} a_{N}^{\prime}(\hat{R}) z(\hat{R})=\int_{0}^{\hat{R}} r^{n-3}\left(\lambda_{1}-\lambda_{N}\right) a_{N} z d r>0 .
$$


But as $a_{N}^{\prime}(\hat{R}) \geqq 0$ and $z(\hat{R}) \leqq 0$, we obtain a contradiction. It follows that $\hat{R}>\tilde{\tilde{R}}(R)$. If we now integrate $(2.20)$ from 0 to $\hat{R}$, we have

$$
\hat{R}^{n-1} a_{N}^{\prime}(\hat{R}) z(\hat{R})=\left(\lambda_{1}-\lambda_{N}\right)\left[\int_{0}^{\tilde{\tilde{R}}(R)} r^{n-3} a_{N} z d r+\int_{\tilde{\tilde{R}}(R)}^{\hat{R}} r^{n-3} a_{N} z d r\right]
$$

Now for small $\left(R_{2}-\bar{R}\right)(c f$. Figure 1$)$, we clearly have both $z(\hat{R})=O\left(R_{2}-\bar{R}\right)$ and

$$
\int_{\tilde{\tilde{R}}(R)}^{\hat{R}} r^{n-1} a_{N} z d r=O\left(R_{2}-\bar{R}\right)
$$

On the other hand

$$
\int_{0}^{\tilde{R}(R)} r^{n-3} a_{N} z d r>0
$$

is not $O\left(R_{2}-\bar{R}\right)$. Thus, for sufficiently small $\left(R_{2}-\bar{R}\right),(2.21)$ gives a contradiction. It follows that $a_{N}(r) \equiv 0$ for $N \geqq 2$ if $\left(R_{2}-\bar{R}\right)$ is sufficiently small. This completes the proof of Theorem 2.2.

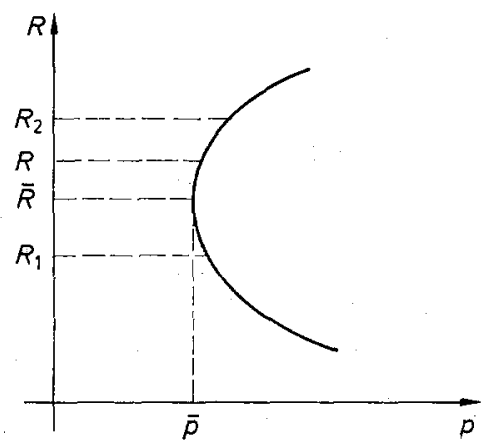

a

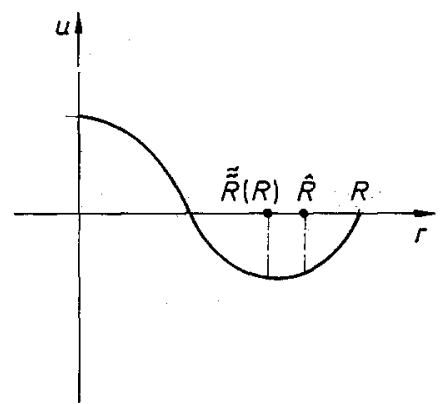

b

Fig. $1 \mathrm{a}$ and $\mathrm{b}$

\section{§ 3. The Existence of Degenerate Radial Solutions for the Perturbed Equation}

We consider first the existence problem for radial solutions of (1.4). Such solutions solve the problem

$$
\begin{gathered}
u^{\prime \prime}+\frac{n-1}{r} u^{\prime}+f(u)+c h(u, r)=0, \quad 0<r<R, \\
u^{\prime}(0)=0=u(R) .
\end{gathered}
$$


We assume in this section that $f$ satisfies conditions (2.2), so that the results of Theorems 2.1, and 2.2 are valid.

We denote by $u_{\varepsilon}(r, p)$ the solution of the initial value problem

$$
\begin{gathered}
u_{\varepsilon}^{\prime \prime}+\frac{n-1}{r} u_{\varepsilon}^{\prime}+f\left(u_{\varepsilon}\right)+\operatorname{ch}\left(u_{\varepsilon}, r\right)=0 \\
u_{\varepsilon}^{\prime}(0)=p>0, \quad u_{\varepsilon}^{\prime}(0)=0 .
\end{gathered}
$$

Associated with this $f$, we have the function $p(R)$ defined in Section 2. For $p=$ $p\left(R_{1}\right)$, we have (from $\left.(2.10)\right), u^{\prime}\left(R_{1}, p\left(R_{1}\right)\right)<0$ and $u\left(R_{1}, p\left(R_{1}\right)\right)=0$. Thus there is an $\eta>0$ such that $u\left(R_{1}+\eta, p\left(R_{1}\right)\right)<0$. Now for $|\varepsilon|$ sufficiently small, solutions of (3.2) are close to solutions of $(2.1)$ on $0 \leqq r \leqq R_{1}+2 \eta$. Hence for $|\varepsilon|$ small, we have $u_{\varepsilon}\left(R_{1}+\eta, p\left(R_{1}\right)\right)<0$ and $u_{\varepsilon}^{\prime}\left(R_{1}+\eta, p\left(R_{1}\right)\right)<0$. It follows that for such $\varepsilon$ there exists a continuous function $R_{1}(\varepsilon)$ satisfying $R_{1}(0)=R$, and

$$
\begin{gathered}
u_{\varepsilon}\left(R_{1}(\varepsilon), p\left(R_{1}\right)\right)=0, \quad u_{\varepsilon}^{\prime}\left(R_{1}(\varepsilon), p\left(R_{1}\right)\right)<0, \\
u_{\varepsilon}\left(r, p\left(R_{1}\right)>0 \quad \text { for } 0 \leqq r_{1}<R_{1}(\varepsilon) ;\right.
\end{gathered}
$$

(cf. Figure 2a). Similarly, for $p=p\left(R_{2}\right)$, we have a continuous function $R_{2}(\varepsilon)$, defined for $|\varepsilon|$ sufficiently small, satisfying $R_{2}(0)=R_{2}$, and continuous functions $\tilde{R}(R), \tilde{\tilde{R}}(R)$ satisfying (cf., Figure $2 \mathrm{~b}$ )

$$
\begin{array}{ll}
u_{\varepsilon}\left(R_{2}(\varepsilon), p\left(R_{2}\right)\right)=0, \quad u_{\varepsilon}^{\prime}\left(R_{2}(\varepsilon), p\left(R_{2}\right)\right)>0, \\
u_{\varepsilon}\left(r, p\left(R_{2}\right)\right)>0 & \text { for } 0 \leqq r<\tilde{R}\left(R_{2}(\varepsilon)\right), \quad u_{\varepsilon}\left(\tilde{R}\left(R_{2}(\varepsilon)\right), p\left(R_{2}\right)\right)=0 \\
u_{\varepsilon}\left(r, p\left(R_{2}\right)\right)<0 & \text { for } \tilde{R}\left(R_{2}(\varepsilon)\right)<r<R_{2}(\varepsilon), \\
u_{\varepsilon}^{\prime}\left(r, p\left(R_{2}\right)\right)<0 & \text { for } 0<r<\tilde{\tilde{R}}\left(R_{2}(\varepsilon)\right), \\
u_{\varepsilon}^{\prime}\left(r, p\left(R_{2}\right)\right)>0 & \text { for } \tilde{\tilde{R}}\left(R_{2}(\varepsilon)\right)<r \leqq R_{2}(\varepsilon) .
\end{array}
$$

Now since $f$ satisfies (2.2), $f$ has a smallest positive root, say $u_{f}$. For $\varepsilon$ small, and $R_{1} \leqq r \leqq R_{2}, f(u)+\varepsilon h(u, r)$ has a root $u_{f}^{\varepsilon}$ near $u_{f}$. For $p$ near $u_{f}^{e}$ the solution $u(r, p)$ does not meet $u=0$, while for large $p$ it meets this line transversally.

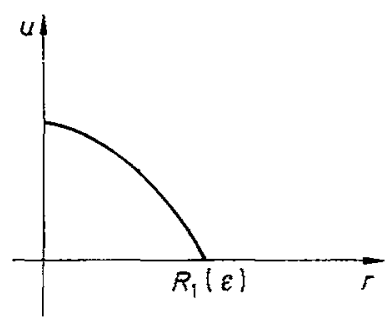

a

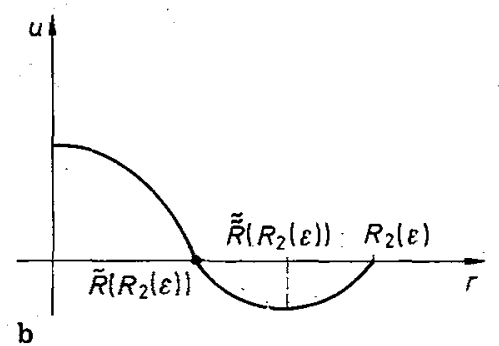

b

Fig. $2 a$ and $b$ 
It follows that there is a continuous function $\bar{R}(\varepsilon), \bar{R}(0)=\bar{R}$, and a point $\vec{p}_{s}$ near $\bar{p}$ (recall $p(\bar{R})=\bar{p})$, such that

$$
\begin{array}{lll}
u_{\varepsilon}\left(r, \bar{p}_{\varepsilon}\right)>0 & \text { for } 0 \leqq r<\bar{R}(\varepsilon), & u_{\varepsilon}\left(\bar{R}(\varepsilon), \bar{p}_{\varepsilon}\right)=0 \\
u_{\varepsilon}^{\prime}\left(r, \bar{p}_{\varepsilon}\right)>0 & \text { for } 0<r<\bar{R}(\varepsilon), & u_{\varepsilon}^{\prime}\left(\bar{R}(\varepsilon), \bar{p}_{\varepsilon}\right)=0 .
\end{array}
$$

This follows from (3.3) and (3.4) since the functions $R_{1}$ and $R_{2}$ are continuous.

Now if $|\varepsilon|$ is sufficiently small, then since $u_{p}(\bar{R}, \bar{p})<0 \quad(c f .(2.4))$, we have

$$
\frac{\partial}{\partial p} u_{\varepsilon}\left(\bar{R}(\varepsilon), \bar{p}_{\varepsilon}\right)<0
$$

This allows us to solve the equation $u_{\varepsilon}(R, p)=0$ for $p$ as a function of $R$, say $p=p_{\varepsilon}(R), R_{1}(\varepsilon) \leqq R \leqq R_{2}(\varepsilon)$; (we are using the same notation as before - this should not cause any confusion). This implies that the solution $u_{\varepsilon}\left(r, p_{\varepsilon}(R)\right)$ of (3.2) is also a solution of (3.1) if $R_{1}(\varepsilon) \leqq R \leqq R_{2}(\varepsilon)$. Thus $u_{\varepsilon}\left(r, p_{\varepsilon}(R)\right)$ also solves (1.4) on this range of $R$. Now as (2.8) and (2.9) followed from (2.4), we have similarly

$$
p_{\varepsilon}^{\prime}(\bar{R}(\varepsilon))=0, \quad \text { and } p_{\varepsilon}^{\prime \prime}(\bar{R}(\varepsilon))>0 .
$$

It is also easy to verify that on the range $R_{1}(\varepsilon) \leqq R<\bar{R}(\varepsilon), u_{\varepsilon}\left(r, p_{\varepsilon}(R)\right)$ enjoys the same properties as $u(r, p(R)), R_{1} \leqq R<\bar{R}$; see (2.11). Similarly, for $\bar{R}(\varepsilon)<$ $R \leqq R_{2}(\varepsilon), u_{\varepsilon}\left(r, p_{\varepsilon}(R)\right)$ satisfies the same properties as $u(r, p(R))$ on $\bar{R}<R \leqq R_{2}$ (cf. (2.12)).

We can now state the following theorem.

Theorem 3.1. If $|\varepsilon|$ is sufficiently small the following hold:

A) $u_{\varepsilon}\left(r, p_{\varepsilon}(R)\right)$ solves $(1.4), \quad R_{1}(\varepsilon) \leqq R \leqq R_{2}(\varepsilon)$

B) There exists a unique continuous function $\hat{R}(\varepsilon)$ near $\vec{R}(\varepsilon)$ such that $u_{\varepsilon}\left(r, p_{\varepsilon}(\hat{R}(\varepsilon))\right)$ is a degenerate solution of $(1.4)$, and $u_{\varepsilon}\left(r, p_{\varepsilon}(R)\right), \quad R \neq \hat{R}(\varepsilon), R_{1}(\varepsilon) \leqq$ $R \leqq R_{2}(\varepsilon)$ is a non-degenerate solution of (1.4).

C) $\hat{R}(\varepsilon)>\bar{R}(\varepsilon)$ if $\varepsilon h_{r}(u, r)<0$, and $\hat{R}(\varepsilon)<R(\varepsilon)$ if $\varepsilon h_{r}(u, r)>0$.

Proof. Part A) has already been proved. Now let $v$ be a solution of the problem

$$
\begin{aligned}
\Delta v+\left[f^{\prime}(u)+\varepsilon h_{u}(u, r)\right] v & =0, \quad x \in D_{R}^{n} \\
v(x)=0, \quad|x| & =R,
\end{aligned}
$$

where $u=u_{\varepsilon}\left(r, p_{\varepsilon}(R)\right)$. We expand $v$ in spherical harmonics:

$$
v=\sum_{N=0}^{\infty} a_{N}(r) \Phi_{N}(\theta), \quad \theta \in S^{n-1}, \quad 0 \leqq r \leqq R,
$$

and we find that $a_{0}(r)$ satisfies

$$
\begin{aligned}
a_{0}^{\prime \prime}+\frac{n-1}{r} a_{0}^{\prime}+\left[f^{\prime}(u)+\varepsilon h_{u}\right] a_{0} & =0, \quad 0<r<R \\
a_{0}^{\prime}(0)=0, \quad a_{0}(R) & =0,
\end{aligned}
$$


and for $N \geqq 1, a_{N}(r)$ satisfies

$$
\begin{gathered}
a_{N}^{\prime \prime}+\frac{n-1}{r} a_{N}^{\prime}+\left[f^{\prime}(u)+\varepsilon h_{u}+\frac{\lambda_{N}}{r^{2}}\right] a_{N}=0, \quad 0<r<R, \\
a_{N}(0)=0=a_{N}(R),
\end{gathered}
$$

where $\lambda_{N}=-N(N+n-2)$, and $u=u_{\varepsilon}\left(r, p_{\varepsilon}(R)\right)$.

Now let

$$
w=\frac{\partial}{\partial p} u_{s}\left(r, p_{\varepsilon}(R)\right)
$$

then $w$ satisfies

$$
\begin{gathered}
w^{\prime \prime}+\frac{n-1}{r} w^{\prime}+\left[f^{\prime}(u)+\varepsilon h_{u}\right] w=0, \quad 0<r<R, \\
w^{\prime}(0)=0, \quad w(0)=1, \quad w(R)<0 .
\end{gathered}
$$

Comparing (3.8) and (3.10) gives $a_{0}(r)=a_{0}(0) w(r)$ so that $0=a_{0}(R)=$ $a_{0}(0) w(R)$ implies $a_{0}(0)=0$, and thus $a_{0}(r) \equiv 0$. Next, for $R_{1}(\varepsilon) \leqq R \leqq R_{2}(\varepsilon)$, let

$$
g_{R}(r)=u_{\varepsilon}^{\prime}\left(r, p_{\varepsilon}(R)\right) .
$$

Then $g_{R}$ satisfies the equation

$$
\begin{gathered}
g_{R}^{\prime \prime}+\frac{n-1}{r} g_{R}^{\prime}+\left[f^{\prime}(u)+\varepsilon h_{u}+\frac{\lambda_{1}}{r^{2}}\right] g_{R}+\varepsilon h_{r}=0 \\
g_{R}(0)=0, \quad g_{R}^{\prime}(0)=u_{\varepsilon}^{\prime \prime}\left(0, p_{\varepsilon}(R)\right)<0,
\end{gathered}
$$

where $u(r)=u_{\varepsilon}\left(r, p_{\varepsilon}(R)\right)$. For $N \geqq 2$, the same arguments as in the proofs of Theorems 2.1 , and 2.2 apply and we obtain $a_{N}(r) \equiv 0$ for $n \geqq 2$.

Thus the degeneracy of $u_{\varepsilon}\left(;, p_{\varepsilon}(R)\right)$ depends on $a_{1}$. Let $a_{1}\left(r, p_{\varepsilon}(R)\right)$ be the solution of the problem

$$
\begin{gathered}
a_{1}^{\prime \prime}+\frac{n-1}{r} a_{1}^{\prime}+\left[f^{\prime}(u)+\varepsilon h_{u}+\frac{\lambda_{1}}{r^{2}}\right] a_{1}=0, \quad 0<r<R, \\
a_{1}(0)=0, \quad a_{1}^{\prime}(0)=u_{\varepsilon}^{\prime \prime}\left(0, p_{\varepsilon}(R)\right) .
\end{gathered}
$$

Then for $R=\bar{R}(\varepsilon)$, we have $g_{\bar{R}(\varepsilon)}(r)<0$ for $0<r<\bar{R}(\varepsilon)$, and $g_{\bar{R}(\varepsilon)}(\bar{R}(\varepsilon))=0$.

Now on $0 \leqq r \leqq R$, as solutions of (3.11) are close to solutions of (3.12), for sufficiently small $|\varepsilon|$, we conclude that for $0 \leqq r \leqq R$

Now by definition

$$
a_{1}\left(r, p_{\varepsilon}(R)\right)-g_{R}(r)=O(\varepsilon) \text {. }
$$

so that

$$
u_{\varepsilon}\left(R, p_{\varepsilon}(R)\right)=0
$$

$$
\frac{\partial u_{s}}{\partial r}\left(R, p_{\varepsilon}(R)\right)+\frac{\partial u_{\varepsilon}}{\partial p}\left(R, p_{\varepsilon}(R)\right) p_{\varepsilon}^{\prime}(R)=0
$$


Then differentiating with respect to $R$ gives

$$
\frac{d}{d R} \frac{\partial u_{\varepsilon}}{\partial r}\left(R, p_{\varepsilon}(R)\right)+\frac{\partial u_{\varepsilon}}{\partial p}\left(R, p_{\varepsilon}(R)\right) p_{\varepsilon}^{\prime \prime}(R)+\frac{d}{d R}\left[\frac{\partial u_{\varepsilon}}{\partial p}\left(R, p_{\varepsilon}(R)\right)\right] \ddot{p}_{\varepsilon}^{\prime}(R)=0
$$

Thus at $R=\bar{R}(\varepsilon),(3.6)$ and (3.7) imply

$$
\left.\frac{d}{d R} \frac{\partial u_{\varepsilon}}{\partial r}\left(R, p_{\varepsilon}(R)\right)\right|_{R=\bar{R}(\varepsilon)}=-\frac{\partial u_{\varepsilon}}{\partial p}\left(R, p_{\varepsilon}(R)\right) p_{\varepsilon}^{\prime \prime}(R)>0
$$

Thus if $R_{1}(\varepsilon)$ and $R_{2}(\varepsilon)$ are near $\bar{R}(\varepsilon)$, then

$$
\frac{d}{d R}\left(g_{R}(R)\right)=\frac{d}{d R} \frac{\partial u_{\varepsilon}}{\partial r}\left(R, p_{\varepsilon}(R)\right)>0 \quad \text { for } R_{1}(\varepsilon) \leqq R \leqq R_{2}(\varepsilon)
$$

Now if $\varepsilon$ is sufficiently small, we have

$$
\frac{d}{d R} a_{1}\left(R, p_{\varepsilon}(R)\right)>0, \quad R_{1}(\varepsilon) \leqq R \leqq R_{2}(\varepsilon) .
$$

From (3.13), we have, for small $\varepsilon$

$$
\begin{aligned}
a_{1}\left(R_{1}(\varepsilon), p_{\varepsilon}\left(R_{1}(\varepsilon)\right)\right) & =g_{R_{1}(\varepsilon)}\left(R_{1}(\varepsilon)\right)+O(\varepsilon) \\
& =\frac{\partial}{\partial r} u_{\varepsilon}\left(R_{2}(\varepsilon), p_{\varepsilon}\left(R_{1}(\varepsilon)\right)\right)+O(\varepsilon) \\
& <0,
\end{aligned}
$$

in view of (3.3). Similarly (3.13) and (3.4) give

$$
\begin{aligned}
a_{1}\left(R_{2}(\varepsilon), p_{\varepsilon}\left(R_{2}(\varepsilon)\right)\right) & =g_{R_{2}(\varepsilon)}\left(R_{2}(\varepsilon)\right)+O(\varepsilon) \\
& =\frac{\partial}{\partial r} u_{\varepsilon}\left(R_{2}(\varepsilon), p_{\varepsilon}\left(R_{2}(\varepsilon)\right)\right)+O(\varepsilon) \\
& >0,
\end{aligned}
$$

Now from (3.14)-(3.16), we see that there exists a unique $\hat{R}(\varepsilon), R_{1}(\varepsilon)<\hat{R}(\varepsilon)<$ $R_{2}(\varepsilon)$ for which $a_{1}\left(\hat{R}(\varepsilon), p_{\varepsilon}(\hat{R}(\varepsilon))\right)=0$. Thus $u_{\varepsilon}\left(r, p_{\varepsilon}(\hat{R}(\varepsilon))\right)$ is a degenerate solution of (3.1), and for $R_{1}(\varepsilon) \leqq R \leqq R_{2}(\varepsilon), R \neq \hat{R}(\varepsilon), a_{1}(r)=0$. Thus statement B) in Theorem 3.1 is proved.

We now consider part C). Thus assume that $\varepsilon h_{r}(u, r)<0$. We shall prove that $\hat{R}(\varepsilon)>\bar{R}(\varepsilon)$. In view of what we have already shown, this is equivalent to proving that $a_{1}\left(\bar{R}(\varepsilon), p_{\varepsilon}(\bar{R}(\varepsilon))\right)<0$. If $a_{1}\left(\bar{R}(\varepsilon), p_{\varepsilon}(\bar{R}(\varepsilon))\right) \geqq 0$, then let $r_{0}$ be the first positive zero of $a_{1}\left(r, p_{\varepsilon}(\bar{R}(\varepsilon))\right)$. Using the same sort of arguments as be- 
fore, we find, from (3.11) and (3.12),

$$
\frac{d}{d r}\left[r^{n-1}\left(g_{R}^{\prime} a_{1}-a_{1}^{\prime} g_{R}\right)\right]=-r^{n-1} \varepsilon h_{r} a_{1} .
$$

Integrating this from $r=0$ to $r=r_{0}$ gives

$$
-r_{0}^{n-1} g_{R}\left(r_{0}\right) a_{1}^{\prime}\left(r_{0}\right)=-\int_{0}^{r_{0}} r^{n-1} \varepsilon h_{r}(u, r) a_{1}(r) d r<0 .
$$

But as $g_{R}\left(r_{0}\right)<0$ and $a_{1}^{\prime}\left(r_{0}\right) \geqq 0$, we arrive at a contradiction. Thus $\hat{R}(\varepsilon)>$ $\bar{R}(\varepsilon)$ if $\varepsilon h_{r}(u, r)<0$. Similarly, we can show that $\hat{R}(\varepsilon)<\bar{R}(\varepsilon)$ if $\varepsilon h_{r}(u, r)>0$. This completes the proof of Theorem 3.1.

\section{§ 4. Symmetry Breaking for Solutions of Equation (1.4)}

We continue to assume that $f$ satisfies hypotheses (2.2). This implies that the results of $\S 2$ and $\S 3$ are valid. In particular Theorem 3.1 is valid, so that $u_{\varepsilon}\left(\dot{r}, p_{\varepsilon}(\hat{R}(\varepsilon))\right)$ is degenerate solution of (1.4). Our goal is to prove that the symmetry actually breaks on this solution. To this end let $I=\left[R_{1}(\varepsilon), R_{2}(\varepsilon)\right]$, and define the operator

by

$$
M:\left\{u \in C^{2}\left(D_{1}^{n}\right): u=0 \text { on } \partial D_{1}^{n}\right\} \times I \rightarrow C^{0}\left(D_{1}^{n}\right)
$$

$$
\begin{aligned}
M(z, R)(x)= & \Delta_{x}\left(z(x)+u_{\varepsilon}\left(|x| R, p_{\varepsilon}(R)\right)\right)+R^{2}\left[f\left(z(x)+u_{\varepsilon}\left(|x| R, p_{\varepsilon}(R)\right)\right)\right. \\
& \left.+\varepsilon h\left(z(x)+u_{\varepsilon}\left(|x| R, p_{\varepsilon}(R)\right),|x| R\right)\right] .
\end{aligned}
$$

From Theorem $3.1 \mathrm{~A}$ ), we know that $M(0, R)=0$ for all $R \in I$. Furthermore, as was shown in Theorem 3.1, the linearized operator about the degenerate solution $\mathcal{u}_{\varepsilon}\left(r, p_{\varepsilon}(\hat{R}(\varepsilon))\right.$ has kernel spanned by $a_{1}\left(r, p_{\varepsilon}(\hat{R}(\varepsilon))\right) \Phi_{1}(\theta)$, where $\Phi_{1}(\theta)$ lies in the eigenspace of the Laplacian on $S^{n-1}$ corresponding to the eigenvalue $\lambda_{1}=-(n-1)$. Hence the equation

$$
0=M_{z}(0, \hat{R}(\varepsilon)) v=\Delta v+\hat{R}^{2}\left[f^{\prime}(u)+\varepsilon h_{u}(u, r)\right] v,
$$

where $u(r)=u_{\varepsilon}\left(r \hat{R}, p_{\varepsilon}(\hat{R}(\varepsilon))\right)$, has an $n$-dimensional solution set spanned by the functions

$$
\frac{x_{i}}{r} a_{1}\left(r \hat{R}, p_{\varepsilon}(\hat{R}(\varepsilon))\right), \quad i=1,2, \ldots, n
$$

(cf. [SW1, 2]). Now as there are no (non-zero) purely radial elements in the kernel, if bifurcation occurs on $u_{\varepsilon}\left(r, p_{\varepsilon}(\hat{R}(\varepsilon))\right)$, then the symmetry breaks.

In order to prove that the symmetry breaks on this solution, we have only to verify the transversality condition (see [SW2])

$$
\int_{D_{1}^{n}} M_{z R}(0, \hat{R}) v^{2}(x) d x \neq 0
$$


for any solution $v$ of (4.1). Now a computation gives

where

$$
\begin{aligned}
M_{z R}(0, \hat{R})= & 2 \hat{R}\left[f_{\varepsilon}^{\prime}(u, r)\right]+\hat{R}^{2}\left[f_{\varepsilon}^{\prime \prime}(u, r)\right]\left[\frac{r}{\hat{R}} \frac{\partial}{\partial r} u\left(r \hat{R}, p_{\varepsilon}(\hat{R})\right)\right. \\
& \left.+p_{\varepsilon}^{\prime}(\hat{R}) u_{p}\left(r \hat{R}, p_{\varepsilon}(\hat{R})\right)\right]+\hat{R}^{2} \varepsilon \frac{d}{d R}\left(h_{u}\right)
\end{aligned}
$$

$$
f_{\varepsilon}^{\prime}(u, r)=f^{\prime}(u)+\varepsilon h_{u}(u, r), \quad \text { and } \quad f_{\varepsilon}^{\prime \prime}(u, r)=f^{\prime \prime}(u)+\varepsilon h_{u u}(u, r) .
$$

As in the appendix of [SW3], we need only prove that

$$
A \equiv \int_{0}^{1} M_{z R}(0, \hat{R}) a_{1}\left(r \hat{R}, p_{\varepsilon}(\hat{R})\right)^{2} r^{n-1} d r \neq 0 .
$$

For notational convenience, we set $u(r) \equiv u_{\varepsilon}\left(r \hat{R}, p_{\varepsilon}(\hat{R})\right)$, and $a(r)=a_{1}\left(r \hat{R}, p_{\varepsilon}(\hat{R})\right)$. Then $a(r)$ satisfies

$$
\begin{gathered}
a^{\prime \prime}+\frac{n-1}{r} a^{\prime}+\hat{R}^{2}\left[f_{\varepsilon}^{\prime}(u, r)\right] a-\frac{n-1}{r^{2}} a=0, \quad 0<r<1, \\
a(0)=0=a(1) .
\end{gathered}
$$

We can now compute the integral in (4.4). To this end note that since $p_{\varepsilon}^{\prime}(\bar{R}(\varepsilon))=0$ and $\hat{R}$ is near $\bar{R}, p_{\varepsilon}^{\prime}((\hat{R}(\varepsilon))=O(\varepsilon)$, so from (4.3),

$$
\begin{aligned}
A & =\int_{0}^{1} M_{z R}(0, \hat{R}) a^{2}(r) r^{n-1} d r \\
& =\int_{0}^{1}\left\{2 \hat{R} f_{\varepsilon}^{\prime}(u, r)+\hat{R}^{2} f_{\varepsilon}^{\prime \prime}(u, r)\left[\frac{r}{\hat{R}} \frac{\partial}{\partial r} u_{\varepsilon}\left(r \hat{R}, p_{\varepsilon}(\hat{R})\right)\right]\right\} a^{2}(r) r^{n-1} d r+O(\varepsilon),
\end{aligned}
$$

where

But

$$
\begin{aligned}
O(\varepsilon)= & \int_{0}^{1} \hat{R}^{2} f_{\varepsilon}^{\prime \prime}(u, r) p_{\varepsilon}^{\prime}(\hat{R}) u_{p}\left(r \hat{R}, p_{\varepsilon}(\hat{R})\right) a^{2}(r) r^{n-1} d r \\
& +\int_{0}^{1} \hat{R}^{2} \varepsilon \frac{d}{d R}\left(h_{u}\right) a^{2}(r) r^{n-1} d r
\end{aligned}
$$

$$
\left\{2 \hat{R} f_{\varepsilon}^{\prime}(u, r)+\hat{R} f_{\varepsilon}^{\prime \prime}(u, r) r \frac{\partial}{d r} u\right\} r=\frac{d}{d r}\left[\hat{R} r^{2} f_{\varepsilon}^{\prime}(u, r)\right]-\hat{R r^{2}} \varepsilon \frac{d}{d r} h_{u},
$$

so that from (4.5)

$$
\begin{aligned}
A & =\int_{0}^{1} \frac{d}{d r}\left[\hat{R} r^{2} f_{\varepsilon}^{\prime}(u, r)\right] a^{2}(r) r^{n-2} d r+O(\varepsilon) \\
& =-\int_{0}^{1} \hat{R r^{2}} f_{\varepsilon}^{\prime}(u, r) \frac{d}{d r}\left(a^{2}(r) r^{n-1}\right) d r+O(\varepsilon) .
\end{aligned}
$$


Using the differential equation in (4.5), we have

$$
\begin{aligned}
A= & \int_{0}^{1} \frac{1}{\hat{R}}\left[a^{\prime \prime}+\frac{n-1}{r} a^{\prime}-\frac{n-1}{r^{2}} a\right]\left[2 a^{\prime} r^{n}+(n-2) r^{n-1} a\right] d r+O(\varepsilon) \\
= & \int_{0}^{1} \frac{1}{\hat{R}}\left\{2 r^{n} a^{\prime \prime} a^{\prime}+(n-2) r^{n-1} a a^{\prime \prime}+2(n-1) r^{n-1}\left(a^{\prime}\right)^{2}\right. \\
& \left.+(n-1)(n-2) r^{n-2} a a^{\prime}-2(n-1) r^{n-2} a^{\prime} a-(n-1)(n-2) r^{n-3} a^{2}\right\} d r \\
& +O(\varepsilon) \\
= & \frac{1}{\hat{R}} \int_{0}^{1}\left\{\frac{d}{d r}\left(r^{n}\left(a^{\prime}\right)^{2}\right)+(n-2) \frac{d}{d r}\left(r^{n-1} a a^{\prime}\right)-(n-1) \frac{d}{d r}\left(r^{n-2} a^{2}\right)\right\} d r+O(\varepsilon) \\
= & \frac{1}{\hat{R}}\left[a^{\prime}(1)\right]^{2}+O(\varepsilon) .
\end{aligned}
$$

But (cf. $(3.10$ or $(3.11))$,

$$
\begin{aligned}
a^{\prime}(1) & =\hat{R} a_{1}^{\prime}\left(\hat{R}(\varepsilon), p_{\varepsilon}(\hat{R}(\varepsilon))\right) \\
& =\hat{R} u_{\varepsilon}^{\prime \prime}\left(\hat{R}(\varepsilon), p_{\varepsilon}(\hat{R}(\varepsilon))\right)+O(\varepsilon) \\
& =\hat{R}(-f(0)-\varepsilon h(0, \hat{R}))+O(\varepsilon)
\end{aligned}
$$

Therefore for small $|\varepsilon|$,

$$
A=\hat{R} f(0)^{2}+O(\varepsilon)>0,
$$

since $f(0)<0$. This proves the transversality condition, and thus the symmetry breaks on $u_{\varepsilon}\left(r, p_{\varepsilon}(\hat{R}(\varepsilon))\right)$. We have thus proved the following theorem.

Theorem 4.1. Let $f$ satisfy (2.2), and for small $|\varepsilon|$ let $u_{\varepsilon}\left(r, p_{\varepsilon}(\hat{R}(\varepsilon))\right)$ denote the degenerate solution of (1.4). Then the symmetry breaks on this solution.

We close with the following remark. Suppose that $\varepsilon h_{r}(u, r)>0$; then as we have seen in Theorem 3.1 C), $\hat{R}(\varepsilon)<\bar{R}(\varepsilon)$. Thus from $(2.10)$ we see that for small $\varepsilon, u_{\varepsilon}^{\prime}\left(\hat{R}(\varepsilon), p_{\varepsilon}(\hat{R}(\varepsilon))\right)<0$. It follows that for $R$ near $\hat{R}(\varepsilon)$, the non-radial solution bifurcating from $u_{\varepsilon}(r, \hat{R}(\varepsilon))$ is positive in $D_{R}^{n}$. In [GNN], it was shown that if $\varepsilon h_{r}(u, r) \leqq 0$, then positive solutions must be radial; thus their result is (in a certain sense) the best possible.

\section{§ 5. An Application to Certain Systems}

We show here how our results can be used to prove the existence of symmetry-breaking solutions for some special systems. 
Consider the system

$$
\begin{aligned}
& \Delta u(x)+g(u(x))=0, \\
& \Delta v(x)+f(v(x))+\varepsilon h(v(x), u(x))=0, \quad x \in D_{R}^{n},
\end{aligned}
$$

together with Dirichlet boundary conditions

$$
u(x)=0=v(x), \quad x \in \partial D_{R}^{n} .
$$

We assume that $f$ satisfies hypotheses (2.2) and that $|\varepsilon|$ is sufficiently small. We further assume that the problem

$$
\begin{gathered}
\Delta u(x)+g(u(x))=0, \quad x \in D_{R}^{n} \\
u(x)=0, \quad x \in \partial D_{R}^{n},
\end{gathered}
$$

admits a radial solution for a range $R \in\left[R_{1}, R_{2}\right]$. In this case the results of $\S 4$ can be applied to (5.1), (5.2). To see this, we let $u(r, R)$ denote a radial solution of (5.3). Then if we use this in the second equation in (5.1), we find that $v$ satisfies

$$
\begin{gathered}
\Delta v(x)+f(u(x))+\operatorname{ch}(v(x), u(|x|, R))=0, \quad x \in D_{R}^{n}, \\
v(x)=0, \quad x \in \partial D_{R}^{n} .
\end{gathered}
$$

This equation is now of the form (1.4), and as the hypotheses of Theorem 4.1 are valid, we see that for sufficiently small $|\varepsilon|,(5.4)$ admits a positive degenerate solution $v_{\varepsilon}\left(r, p_{\varepsilon}(\hat{R}(\varepsilon))\right)$ on which the symmetry breaks. Thus there is symmetry breaking for the system (5.1) on the solution $\left[u(r, \hat{R}(\varepsilon)), v_{\varepsilon}\left(r, p_{\varepsilon}(\hat{R}(\varepsilon))\right)\right]$.

Acknowledgment. This research was supported in part by National Science Council of Taiwan, R.O.C., and in part by the NSF under Grant No. MCS, 830123 and by the ONR under Grant No. N00014-88-K-0082.

\section{References}

[GNN] Gidas, B., W. M. NI, \& L. Nirenberg, Symmetry of positive solutions of nonlinear elliptic equations in $R^{n}$, Comm. Math. Phys. 68, (1979), 209-243.

[SW1] Smoller, J., \& A. Wasserman, Existence, uniqueness, and nondegeneracy of positive solutions of semilinear elliptic equations, Comm. Math. Phys. 95, (1984), 129-159.

[SW2] SMOLcer, J., \& A. WASSERMAN, Symmetry-breaking for positive solutions of semilinear elliptic equations, Arch. Rational Mech. Anal. 95, (1986), 217-225.

[SW3] SMOlLer, J., \& A. WASSERman, Bifurcation from symmetry, Nonlinear Diffusion Equations and Their Equilibrium States, II (ed. by W.-M. NI, L. A. PeleTIER, \& J. Serrin), Springer-Verlag, New York, 1988, 273-288.

\section{National Tsing Hua University \\ Hsinchu, Taiwan \\ and \\ University of Michigan \\ Ann Arbor.}

